premature ventricular beats of ex vivo heart, under the acute adrenergic challenge, they significantly enhanced the frequency of premature ventricular beats.

Conclusions BPA promotes arrhythmogenesis in female rat heart by induced DADs, and effects of BPA and $E_{2}$ are synergistic instead of additive.

\section{e0181 RENALASE DEFICIENCY IN HEART FAILURE-A NOVEL MECHANISM UNDERLYING CIRCULATING NOREPINEPHRINE ACCUMULATION}

doi:10.1136/hrt.2010.208967.181

Gu Rong, Lu Wen, Xie Jun. Department of Cardiology, Affiliated Drum Tower Hospital, Nanjing University Medical School, Nanjing, China

Background Sympathetic overactivity and catecholamine accumulation are important characteristic findings in heart failure, which contribute to its pathophysiology. However, the mechanism underlying circulating catecholamine accumulation remains largely unclear.

Objective To identify a novel mechanism underlying norepinephrine accumulation in a rat model of heart failure.

Methods and results Initially, we constructed a rat model of unilateral renal artery stenosis and found that the expression of renalase, a previously identified secreted amine oxidase, was markedly reduced in the ischaemic compared to the non-ischaemic kidney. Subsequently, we utilised an isolated perfused rat kidney model to demonstrate that the clearance rate of norepinephrine decreased with reduction of either perfusion flow or pressure. On the basis of these findings, we hypothesised that the reduced renal blood supply which occurs in heart failure would result in impaired synthesis of renalase by the kidney and consequently reduced degradation of circulating norepinephrine. To verify this, we used a rat model of infarction-induced heart failure caused by ligation of the left anterior descending coronary artery. In these rats, renal expression of renalase, when measured at 4 weeks, was reduced, and this was associated with an increase in circulating norepinephrine. Conclusions We conclude that impaired synthesis of renalase by the kidney may represent a novel mechanism underlying circulating norepinephrine accumulation in heart failure.

\section{e0182 ELECTROPHYSIOLOGICAL SUBATRATE FOR CANINE ATRIUM}

doi:10.1136/hrt.2010.208967.182

Liu Ying, Yang Yanzong, Jiang Yinong, Xia Yunlong, Gao Lianjun, Yang Donghui, Li Shijun, Yin Xiaomeng, Lin Zhihu. The First Affiliated Hospital of Dalian Medical University, Dalian, China

Objective Hypertension is frequently complicated by atrial fibrillation (AF). However, the atrial substrate for AF is not known. This study investigated the electrophysiological properties of atrial repolarisation by monophasic action potential (MAP) in order to explore the mechanism of paroxysmal AF initiation and maintenance.

Methods MAP were recorded from left and right atrium in 14 canine. action potential duration (APD) at 90\% repolarisation $\left(A P D_{90}\right)$, Repetitive atrial firing (RAF, the occurrence of two or more successive premature atrial activations with return cycle of $250 \mathrm{msec}$ or less following atrial stimulation) and APD alternans (the difference in APD between two consecutive beats, were induced by overdrive pacing at LA and RA) were induced by use of programmed stimulation at LA and RA. In the study, episodes of PAF were recorded and analysed.

Results $\mathrm{APD}_{90}$ were significantly shorter in the left atrium compared to the right atrium $((157.4 \pm 43.5)$ vs $(170.9 \pm 37.9)$, $\mathrm{p}<0.05)$ ). The mean $\mathrm{S}_{1} \mathrm{~S}_{2}$ interval induced RAF was $(130 \pm 32) \mathrm{ms} .15$ RAF were induced in 14 dogs. RAF induced in LA were more than in RA (11 vs $4, p<0.05)$. Alternans of APD were induced at CL of $(162 \pm 25)$ ms. 13 APD alternans were induced at LA (8) and RA (5) of 14 dogs. In total, 61 episodes of PAF were induced in 14 canines. 38 episodes of PAF were induced in the left atrium, more than in the right atrium $(23, \mathrm{p}<0.05)$.

Conclusions The incidence of RAF and alternans was significantly higher in LA than in RA. Heterogeneity between LA and RA repolarisation creates subatrate for re-entrant arrhythmias and vulnerability to atrial fibrillation.

\section{E0183 LIVIN PROTECTS AGAINST CARDIOMYOCYTE APOPTOSIS IN ANOXIA/REOXYGENATION INJURY VIA P38-MEDIATED SIGNAL PATHWAY}

doi:10.1136/hrt.2010.208967.183

${ }^{1}$ Chen Ling, ${ }^{1}$ Hong Kui, ${ }^{1} \mathrm{Hu}$ Jianxin, ${ }^{2}$ Yin Xihu, ${ }^{2}$ Luo Yun. ${ }^{1}$ The Second Affiliated Hospital of Nanchang University, Nanchang, China; ${ }^{2}$ The First People's Hospital Jiujiang, Jiujiang, China

Introduction Although anoxic preconditioning (APC) in the myocardium has been investigated for many years, its physiological mechanism is still not completely understood. Increasing evidence indicates that transiently increased resistance to ischaemic damage following APC is dependent on de novo protein synthesis. However the key effector pathway(s) associated with APC still remains unclear. Livin, a member of the inhibitor of apoptosis protein (IAP) family, since IAP-mediated activation of JNK1, as well as protection against TNF- $\beta$ and ICE-induced apoptosis. The detailed mechanism underlying its antiapoptotic function in cardiomyocytes has not yet been fully characterised.

Objective To investigate whether Linvin expression might be aberrantly induced in cardiomyocytes that were subjected to anoxia/ reoxygenation $(A / R)$ injury and to investigate whether Linvin might also contribute to cardio-protection after APC.

Methods We cloned a Linvin expression vector, transfected it into rat cardiomyocytes, and examined Linvin expression in rat cardiomyocytes that were subjected to A/R injury. Moreover, we studied the role of three major MAPK pathways, for example, p38 MAPK, JNK, and ERK1/2, in order to evaluate the molecular mechanism underlying Linvin up-regulation and $\mathrm{A} / \mathrm{R}$ induced cardiomyocyte injury. Results APC induced an up-regulation of Linvin and the transfection of Linvin gene into the cardiomyocytes attenuated A/R injury. The inhibition of p38 MAPK by SB203580 abolished both the Linvin up-regulation and the cardio-protection provided by APC.

Conclusion APC could act to protect the heart from A/R injury with cooperation from the Linvin in addition, it up-regulates Linvin expression through a p38 MAPK signalling pathway.

\section{e0184 THE PROTECTION EFFECTS OF TRIMETAZIDINE ON RATS MYOCARDIAL INFRACTION}

doi:10.1136/hrt.2010.208967.184

${ }^{1}$ Yanting Luo, ${ }^{1}$ Jinlai Liu, ${ }^{2}$ Fei Chen, ${ }^{2}$ Wen Tan. ${ }^{1}$ Department of Cardiology, The Third Affiliated Hospital, Sun Yat-sen University, Guangzhou, China; ${ }^{2}$ Key-Pharma Biomedical Company, Dongguan, P. R. China

Objective To observe the myocardial protection effects of trimetazidine on Sprague-Dawley (SD) rats with myocardial infarctions (MI).

Methods 90 SD rats were randomly assigned to normal control group $(\mathrm{NL}, \mathrm{n}=30)$, Trimetazidine group $(\mathrm{T}, \mathrm{n}=30)$ and sham-operated group 